\title{
RAPID SPECTROSCOPIC AND PHOTOMETRIC \\ VARIABILITY IN THE Be STAR ALPHA ERIDANI
}

\author{
N.V.LEISTER,E.JANOT-PACHECO,M.T.C.BUCK,M.P.DIAS \\ Depto. Astronomia, IAG-USP, Brazil \\ and
}
A.M.HUBERT,H.HUBERT,M.FLOQUET and D.BRIOT
Dasgal, Obs. de Paris-Meudon, France

\section{Introduction: $\alpha$ Eri a variable Be star}

We present here a preliminary report on the analysis of simultaneous photometric and spectroscopic observations of the Be star $\alpha$ Eri, taken during 1992 at the Laboratório Nacional de Astrofísica. $\alpha$ Eri (Archenar, HR472, HD10144, B3-4 III-IV) is the brightest Be star in the sky. It shows activity cycles in a time scale of a few years. Balona et al. (1987) presented a comprehensive study of the star based on photometric and spectroscopic observations. They found a periodicity of 1.26 days in both radial velocity and light variations and argued that a spotted star rotating at this period is the simplest working model that explains the observations.

\section{Observations, Reductions and Discussion}

We obtained about 110 high resolution, high signal-to-noise spectra of $\alpha$ Eri in 1992 July 14,15 and 16 at the L.N.A. with a EMI CCD camera attached to the coudé spectrograph of the $1.60 \mathrm{~m}$ telescope. Simultaneous photometric observations through the Strömgrem $b$ filter were made with the $0.60 \mathrm{~m}$ Zeiss reflector on the same site. Spectra were reduced at Instituto Astronômico e Geofísico, Universidade de São Paulo using the "eVe" package. Period search in photometric data were performed through standard FFT and DFT algorithms.

Moving bumps are clearly seen in the residuals (individual spectra minus mean profile).We get $l=|m|=10 \pm 2$ for $\alpha$ Eri in July 1992. The travelling period of bumps is $P_{\text {bumps }}=2 \pi \mathrm{V} \sin i / \mathrm{a}$, which gives in the present case $P_{\text {bumps }}=1.5 \pm 0.3$ days. If the rotational period of the star is 1.26 days (see above), then the NRP are retrograde. The stellar surface pattern due to NRP is reproduced around a star with a periodicity $P_{p}=P_{b u m p s} / m$, which in our case is $0.15 \pm 0.06 \mathrm{~d}$. Small brightness variations due to this changing surface pattern are expected to occur (e.g. Balona 1987). They should show up in the simultaneous photometric data that will be modulated 
with a period $P_{p}$ (see below). Moving bumps can be followed in our July 1992 spectra over a range $\sim \pm 350 \mathrm{kms}^{-1}$ which is considerably larger than $V \sin i\left(225 \mathrm{kms}^{-1}\right.$, Slettebak 1982) for $\alpha$ Eri. This is clearly seen in the "mean absolute deviation" of the spectra (Walker 1991): the region where line profile variability occur is quite evident and extends from about -300 $\mathrm{kms}^{-1}$ up to $350 \mathrm{kms}^{-1}$. This kind of phenomenon has also been reported in other Be stars (e.g. Floquet et al. 1992) and deserves further investigation, as it can be an important clue to the Be phenomenon.

Photometric data obtained in 1992 July 14,15 and 16 have been analysed through Fourier transform techniques to search for periodicities. $P_{p}=0.15$ $\pm 0.06 \mathrm{~d}$ deduced from the spectral analysis corresponds to a frequency $\nu_{p}=6.7 \pm 2.4 \mathrm{c} / \mathrm{d}$. Unfortunately, the time span of the observations for each night is less than about four hours. It is thus not possible to find the above frequency. However, the periodogram shows peaks at multiples of this (supposedly fundamental) frequency around $13,20,23$ and $33 \mathrm{c} / \mathrm{d}$, with $85 \%$ success probability for the $F$ distribution for the ensemble of them.

\section{Conclusions}

Spectral analysis of $\alpha$ Eri suggests the presence of nonradial pulsations with $l=|m|=10 \pm 2$ in July 1992 . This could be the result of the interference of various modes: they cannot be easily distinguished with the kind of analysis presented here. Simultaneous photometric data shows brightness oscillations at frequencies compatible with the first four harmonics of the above determined NRP. However, the power spectrum resolution fails to establish precisely the frequency values and the number of pulsations present in the star. A more extensive analysis of $\alpha$ Eri based on observations spanning about two years will be presented elsewhere.

\section{Acknowledgements}

This research is supported in part by FAPESP and CNPq (Brazil), and by CNRS (France).

\section{References}

Balona L.A.: 1987, M.N 224, 41

Floquet M., Hubert A.M., Janot-Pacheco E., Mekkas A., Hubert H. and Leister N.V.: 1991, $A \& A$ 264, 177

Slettebak A.: 1982, ApJS 50, 55

Walker G.A.H.: 1991, Rapid Variability of $O B$ Stars: Nature and Diagnostic Value, ESO workshop, ed. D. Baade, 27 